- Editorial

\title{
Irritable Bowel Syndrome and Synbiotics
}

\author{
Soo Young Kim* \\ Department of Family Medicine, Kangdong Sacred Heart Hospital, Hallym University College of Medicine, Seoul, Korea
}

\section{See original paper on 2}

Irritable bowel syndrome (IBS) is a functional bowel disorder characterized by abdominal pain, abdominal bloating, and altered bowel habits. ${ }^{1)}$ There are several pharmacological therapies for treating IBS, but none have been demonstrated to alter the natural history of IBS in the long term. In this regard, attention has been focused on controlling symptoms by inducing changes in intestinal bacteria. Drugs used for such purposes include prebiotics and probiotics. Probiotics are living bacteria known to be beneficial to human health, and prebiotics are ingredients in foods, such as fructooligosaccharides or inulin, that help the beneficial bacteria grow. The combination of prebiotics and probiotics is called synbiotics. According to a recently published systematic review, ${ }^{2)}$ the amount of evidence for the therapeutic effect of IBS in these products has been inconsistent. Most evidence is limited to probiotics.

In the present issue, Lee et al. ${ }^{3)}$ investigated the dose-response effects of synbiotic supplementation on bowel symptoms and fatigue in patients with IBS. Thirty patients with IBS were randomly assigned to three group (high-dose of synbiotics, low-dose of synbiotics, and placebo) and received treatment for 8 weeks. The authors found no significant dose-dependent clinical effects of synbiotics in patients with IBS. However, significant positive effects of high-dose synbiotics on gastrointestinal symptoms and fatigue were observed in patients with IBS.

This study is meaningful because the authors investigated whether synbiotics exerted synergistic beneficial effects to help resolve fatigue in patients with IBS. However, additional evidence may be needed because of the small sample and effect sizes in this study.

\section{CONFLICT OF INTEREST}

No potential conflict of interest relevant to this article was reported.

\section{REFERENCES}

1. Lovell RM, Ford AC. Global prevalence of and risk factors for irritable bowel syndrome: a meta-analysis. Clin Gastroenterol Hepatol 2012;10:712-21.

2. Ford AC, Quigley EM, Lacy BE, Lembo AJ, Saito YA, Schiller LR, et al. Efficacy of prebiotics, probiotics, and synbiotics in irritable bowel syndrome and chronic idiopathic constipation: systematic review and meta-analysis. Am J Gastroenterol 2014;109:1547-61.

3. Lee SH, Cho DY, Lee SH, Han KS, Yang SW, Kim JH, et al. A randomized clinical trial of synbiotics in irritable bowel syndrome: dose-dependent effects on gastrointestinal symptoms and fatigue. Korean J Fam Med 2019;40:2-8. 